\title{
Posterior SMA Syndrome following subcortical stroke: Contralateral akinesia reversed by visual feedback
}

\author{
Narges Radman ${ }^{\mathrm{a}, *}$, Stephanie Cacioppo ${ }^{\mathrm{b}}$, Lucas Spierer ${ }^{\mathrm{a}}$, Eric Schmidlin ${ }^{\mathrm{c}}$, \\ Eugène Mayer ${ }^{\mathrm{d}}$, Jean-Marie Annoni ${ }^{\mathrm{a}, \mathrm{d}}$ \\ a Neurology Unit, Medicine Department, University of Fribourg, Fribourg 1700, Switzerland \\ ${ }^{\mathrm{b}}$ High Performance Electrical Neuroimaging Laboratory, CCSN, Department of Psychology, University of Chicago, Chicago, IL, USA \\ c Physiology Unit, Medicine Department, University of Fribourg, Fribourg, Switzerland \\ ${ }^{\mathrm{d}}$ Department of Neurology, Geneva University Hospital, Geneva, Switzerland
}

\begin{abstract}
Background: The supplementary motor area (SMA) plays a key role in motor programming and production and is involved in internally-cued movements. In neurological populations, SMA syndrome following a lesion to the "SMA proper" is characterized by transient impairment of voluntary movements and motor sequences. This syndrome is assumed to follow on from an interruption of the motor corticosubcortical loop, and some case reports indicate that such a syndrome could occur after a brain lesion isolating the SMA from subcortical structures.

Aim: To characterize the pattern of motor impairments in a patient whose stroke disconnects the SMA from the subcortical motor loop.

Method: A patient developed a moderate transient left hemiparesis following a subcortical stroke in the right anterior cerebral artery area, which disconnected the SMA from basal ganglia. Eight days after the stroke, when the hemiparesis had regressed, the patient presented a specific SMA motor disorder of the left hand which manifested as an akinesia and was exacerbated when his visual attention was not directed towards his hand. We assessed finger tapping with left and right hands, eyes closed and open, in the left and right hemispace. We indexed movement speed as the number of taps filmed over 5-s periods.

Results: Left motor weakness (grasping strength of right hand: $49 \mathrm{~kg}$ and left hand: $41 \mathrm{~kg}$ ) was resolved in a week. Ideomotor and ideational gestures and motor sequences were preserved. On the tapping task, left-hand tapping was slower than right-hand tapping. Critically, visual feedback improved tapping speed for the left, but not for the right, hand. The hemispace of the task execution had no effect on tapping performance.

Conclusion: Our results suggest that SMA-basal ganglia disconnection decreases contralateral movement initiation and maintenance and this effect is partly compensated by visual cues.
\end{abstract}

\section{Introduction}

The supplementary motor area (SMA) - a region located within each cerebral hemisphere at the posterior medial border of the frontal lobe - has been shown to play a key role in various aspects of motor planning, readiness for action, movement initiation, speed and even execution (Roland et al., 1980; Jenkins et al., 1992; Orgogozo \& Larsen, 1979), as well as unconscious motor activation or inhibition (D'Ostilio et al., 2012). Non-human primate (NHP)

\footnotetext{
* Corresponding author. Tel.: +41264268151.

E-mail addresses: narges.radman@unifr.ch, narges.radman@gmail.com (N. Radman), cacioppos@uchicago.edu (S. Cacioppo). Lucas.spierer@unifr.ch (L. Spierer), eric.schmidlin@unifr.ch (E. Schmidlin), jean-marie.annoni@unifr.ch (J.-M. Annoni).
}

studies showed the direct functional involvement of the SMA in muscles involved in manual dexterity (Boudrias et al., 2010; Maier et al., 2002).

Previous literature further indicates that the SMA is involved in the "temporal" organization of sequential hand movements or, more generally, in the initiation of movements which have to be performed according to a precise timing plan (Cunnington, Bradshaw, \& Iansek, 1996; Halsband et al., 1992). Single unit recordings in animal models/ non-human primates also demonstrated that SMA encodes hand motion speed and direction (Boudrias et al., 2006). It has been proposed that the SMA is composed of two anatomo-functionally distinct areas: the pre-SMA (divided into anterior pre-SMA and posterior pre-SMA) and the SMA or "SMA proper" (He, Dum, \& Strick, 1995; Luppino et al., 1993a, b). Although controversial, the "SMA proper" is thought to be involved in repetitive movements, 
while the pre-SMA may be involved in the production of sequential movements (melokinetic activity) (Luppino et al., 1993a, b; Catalan et al., 1998).

Lesion and activation studies suggest that the SMA complex is primarily involved in internally-cued rather than externally-cued movements (Jenkins et al., 1992; Cunnington et al., 1996; Jahanshahi et al., 1995; Okano, 1992). In particular, the pre-SMA is involved in internally-guided movements in the absence of visual feedback (Ogawa, Inui, \& Sugio, 2006), though recent evidence suggest that the SMA, particularly the more caudal SMA proper is involved in both internally and externally cued movements (Cunnington et al., 2002).

The major input to the SMA ultimately originates from the basal ganglia (BG); (dorsal putamen to posterior SMA and dorsal caudate to anterior SMA) via the thalamo-cortical pathway (Cunnington et al., 1996; Marsden, 1989; Catalan et al., 1999). Due to the strong association between the SMA and the BG, disconnection of BG output to the SMA produces Parkinsonian symptoms (Cunnington et al., 1996; Kim, 2001) and impairment in the initiation and rapidity of movements, namely, akinesia. There is little direct connectivity between motor areas and the subcortical visual pathways, such as superior colliculi (at least in primates (Fries, 1985)). However, connectivity analysis also reports reciprocal connections between SMA and visual areas (Herrero, Barcia, \& Navarro, 2002).

In clinical settings, SMA syndrome, a consequence of surgical resection of the SMA, is characterized by a transient hemiparesis or motor apraxia with variable degrees of speech arrest followed by rapid recovery of neurological impairments (Kasasbeh et al., 2012). Bannur and Rajshekhar (2000) found a delay in movement initiation and spasticity in three out of six patients with SMA resection. Other case reports emphasize the motor slowness associated with SMA lesion or SMA disconnection from BG following cerebrovascular accidents (CVA) (Kim, 2001; Gentilucci

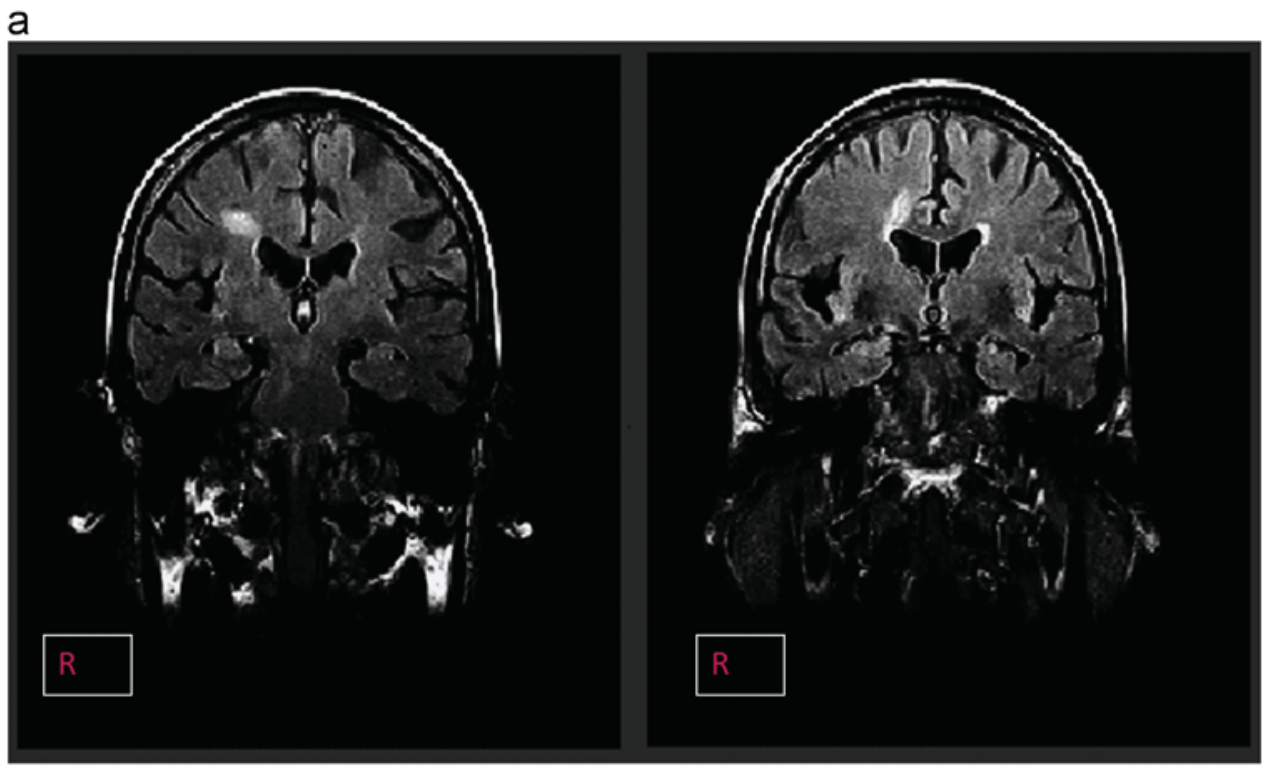

b
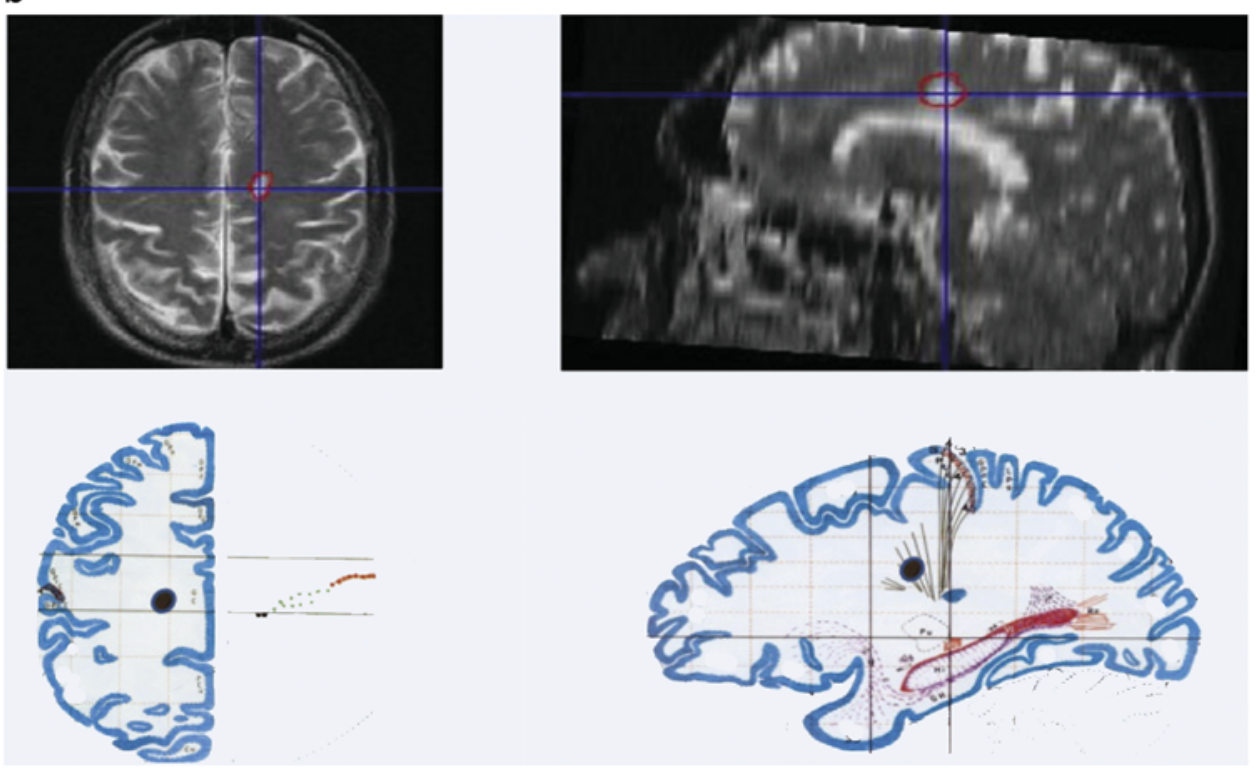

Fig. 1. (a) Patient's MRI coronal section; lesion creates a disconnection between right posterior SMA and caudate/putamen. (b) Patients lesion, in axial plate and reconstruction in the sagittal plane. This subcortical lesion disconnects the posterior SMA from the basal ganglia. 
et al., 2000). All these clinical descriptions suggest a certain degree of akinesia. To date, there are, however, no case studies testing the sensitivity of this motor impairment to visual cues.

In this study, we present a clinical case of transitory motor akinesia following a subcortical infarct in the white matter between the SMA and the striatum disconnecting the SMA from the BG. Based on previous evidence for the involvement of the SMA in internally-guided movements in the absence of visual feedback, we tested whether visual feedback could compensate for the SMA in disconnection-induced akinesia.

\section{Material and methods}

\subsection{Clinical case}

GC, a 69 year-old right-handed male patient, was admitted to hospital for a sudden moderate left-sided hemiparesis, predominating in his arm. Hemiparesis symptoms occurred five days before the hospitalization as ascertained by the patient's self-report. The motor deficit recovered almost completely within $48 \mathrm{~h}$ after hospital admission. However, one week after the stroke, the patient complained of an inability to move his left hand correctly, as if it was "stuck in one position despite the fact that the patient was feeling it correctly". According to the patient, "to command [his] left hand to start a movement, [he] had to look at the fingers and concentrate on the movement, as if [he] wanted to hypnotize his hand. Moreover, [he] has noted slowness in the left-hand movements".

\subsection{Neurological evaluation}

The neurological evaluation, one week after the stroke, was characterized by a slight brachial paresis, scored as 5-(based on 0 to 5 Medical Research Council scale for muscle strength) (Council, 1976), with a left grasping strength of $41 \mathrm{~kg}$ and a right grasping strength of $49 \mathrm{~kg}$ (based on the $10 \%$ rule for effect of handedness on grasp strength, this difference was not related to handedness (Bohannon, 2003)) and a left motor akinesia, characterized by slowness in both initiation and maintenance of movement. Clinically, this akinesia was also characterized by an irregularity in repetitive movements such as in finger-tapping tasks. When GC executed the movement using visual control and external auditory cues (for example, when he was required to tap his finger in synchrony with a metronome), the movements were better initiated and more easily and regularly executed. The rest of neurological evaluation was within the normal range, including cranial nerve, muscle tone, deep tendon reflexes, sensory evaluation (pain, position and vibration sense, stereo gnosis), and coordination. Walking was normal except for a slight decrease in accompanied balance of the upper limb.

A magnetic resonance imaging (MRI) acquisition organized three days after admission showed a focal subcortical lesion of the posterior part of the superior prefrontal areas, deep in the right SMA, with damage to the motor cortical subcortical loop, particularly between BG and SMA (Fig. 1).

\subsection{Neuropsychological evaluation}

The neuropsychological evaluation, one week after the stroke, showed a cooperative and oriented patient with no cognitive impairment. Language was flawless and writing was preserved. Long-term memory, as assessed with the Rey auditory-verbal learning test and the Rey visual design learning test, was also preserved. Buccofacial and constructional praxis was unimpaired. No visual agnosia was visible in the Poppelreuter test and the knowledge of body parts was normal. Tactile gnosis and haptic abilities were perfect (6/6 objects recognized with both hands). There was neither astereognosia nor signs of alien hand syndrome (Table 1).

Specific testing for gestures (see Table 2) showed no deficit in either imitation or verbal command. There was only one hesitation in the military salute with the left hand, but this was corrected by visual inspection. All other movements were correct with both hands (notably slower with the left hand). Luria gestural sequences were slow with the left hand but without perseveration. In conclusion, except for the slowness, there was neither ideomotor nor melokinetic apraxia.

\subsection{Assessment of motor akinesia}

In order to further determine the effect of SMA disconnection in the patient's movement initiation, we investigated his motor akinesia under different conditions by analysing his pattern of finger tapping with right and left hands, and performed a finger-tapping experiment with and without visual feedback in the left or right hemispace (with respect to the patient's body midline)
Table 1

Initial neuropsychological evaluation of GC, 8 days after the stroke. Boston naming (French version), verbal fluency, gestural praxis, RAVLT (Rey's auditory verbal learning test) and ROCF (Rey-Osterrieth complex figure test) are part of the usual evaluation used in the stroke unit. N: Normal. LN: Lower Normal.

\begin{tabular}{lll}
\hline Language & Spontaneous speech & $\begin{array}{l}\text { N, presence of two } \\
\text { semantic approximations }\end{array}$ \\
\hline & Boston naming & $\mathrm{N}$ \\
& Verbal semantic & $\mathrm{N}$ \\
& Verbal phonological fluency & $\mathrm{N}$ \\
Calculation & Comprehension & $\mathrm{N}$ \\
Praxis & Oral and written & $5 / 5(\mathrm{~N})$ \\
& Symbolic gestures & $3 / 3(\mathrm{~N})$ \\
Gnosis & meaningless gestures & $3 / 3$ \\
& Embedded figures & $2 / 2$ \\
& Line bisection & $5 / 5(\mathrm{~N})$ \\
& Finger recognition on tactile & $\mathrm{N}$ \\
Executive & stimulation & $5 / 5(n)$ \\
function & Stroop & $\mathrm{LN}$ \\
Memory & Trail making test & $\mathrm{N}$ \\
& Digit span & $\mathrm{N}$ \\
& Visuo-spatial span & $\mathrm{N}$ \\
& RAVLT & $\mathrm{N}$ \\
& ROCF & $\mathrm{N}$ \\
\hline
\end{tabular}

Table 2

The following pantomimes were used to test praxis: three symbolic gestures (military salute, good bye, saying "no" to a baby), Comb, using a toothbrush, hit a nail with a hammer, and two non-sense gestures. Only pantomimes were tested in verbal command.

\begin{tabular}{lllll}
\hline & Closed eyes & \multicolumn{3}{c}{ Open eyes } \\
\hline Left hand & + & + & + & + \\
Right hand & + & + & + & + \\
\hline
\end{tabular}

Symbolic gestures and pantomime of tool use.

\subsection{Experimental design}

We used a $2 \times 2 \times 2$ design with hands (left vs. right), hemispace (left vs. right) and visual feedback (with vs. without) as within-subject factors, enabling us to control for motor neglect as well as for visual feedback.

\subsection{Patient's instruction}

The patient was instructed to tap his index finger to his thumb repeatedly, regularly, as fast as possible and with the largest amplitude as possible during a 20 -s period. In order to ensure pure distal movements, the medial part of the hand was placed on the table during the task (Fig. 2). This assessment was performed in two consecutive days and each day contained three similarly-ordered sessions.

The order of the 20-s tapping task execution consisted of a sequence of four conditions: I. Right hand-right hemispace, II. Right hand-left hemispace, III. Left hand-left hemispace, IV. Left hand-right hemispace. In the first day of the assessment, these tasks have been performed in a series of $A B-A B-A B$, in which, (A) the above mentioned sequence of four conditions with open eyes then, (B) the above mentioned sequence of four conditions with closed eyes. The same order was respected for the second day.

\subsection{Measures}

Tapping performance and movement times were recorded.

\subsection{Tapping performance recordings}

A video recording was taken throughout the sessions using an invariant camera setting and a fixed distance $(1.5 \mathrm{~m})$ between the camera and the patient's hands.

\subsection{Tapping speed recordings}

Tapping speed was measured by counting the number of taps per fixed 5-s period. During the assessment, we observed that the patient has a slowed initiation. 


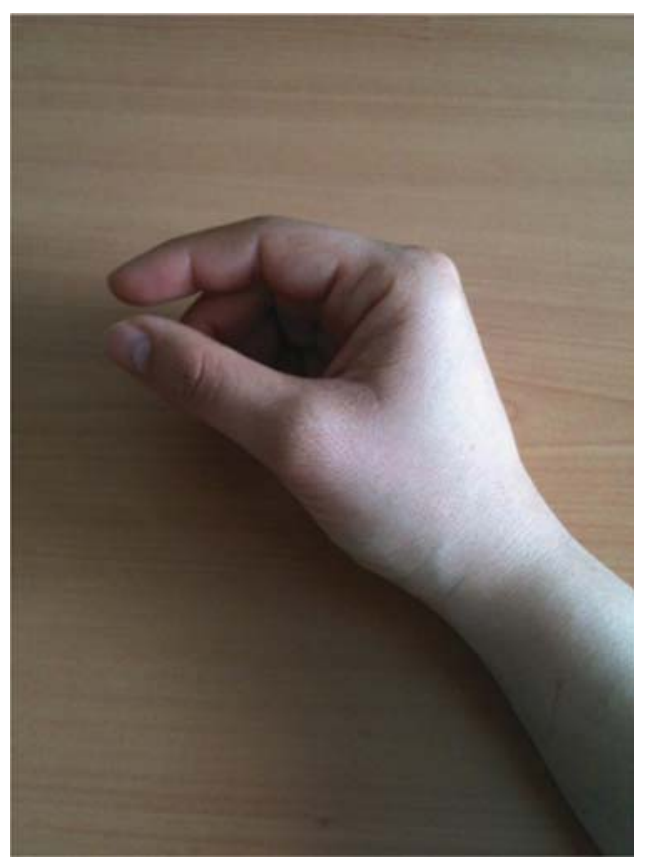

Fig. 2. Position of the hand during the tapping task. Note that tapping speed was similar when the patient had his forearm in the vertical position.

Therefore, in order to reduce the effect of this part on the results and to be sure of a steady state, we performed a separated analyses on the initial part of tapping (the first $5 \mathrm{~s}$ of each session) and maintenance of tapping in the remaining $15 \mathrm{~s}(3 \times 5 \mathrm{~s})$. These measures were obtained using image processing and movie editing programs; Image (http://rsbweb.nih.gov/ij/) Virtual Dub-1.9.11 (http://www.virtualdub.org/) and MAGIX movie edit pro 2013 with a time resolution of $40 \mathrm{~ms}$ for the images that has been taken (http://www.magix.com/us/movie-edit-pro/).

2.10. Tapping analyses

As mentioned above, in order to have pure results on the initiation and maintenance speed, the first 5-s period of each condition was separated from the other three 5-s periods. Initiation speed (the number of taps in the first 5-s) was analyzed using a two-way ANOVA with hand (left and right) and visual feedback (open vs closed eyes) as factors. Tapping maintenance speed (the number of taps per 5-s period) was submitted to a three-way ANOVA with hemispace (left vs. right), hand (left vs. right) and visual feedback (with vs. without) as factors. In both analyses, 5-s periods were used as repeated measures and the variance on number of taps across these periods was reported.

\section{Results}

The patient has a slowed initiation and maintenance speed with left hand while the eyes were closed, which was measured as a lower number of tapping per 5-s segments.

\subsection{Tapping speed}

There was a main effect of factor Hand, i.e., faster right than left hand tapping $\left(f(1,136)=457.4 ; p<0.001 ; h_{p}^{2}=0.8\right)$, a main effect of factor visual feedback, i.e., faster tapping with than without visual feedback $\left(f(1136)=28.9 ; p<0.001 ; h_{p}^{2}=0.2\right)$, and a significant hand $\times$ visual feedback interaction. i.e., more improvement of tapping performance with visual feedback with the left than right hand $\left(f(1,136)=24.6 ; p<0.001 ; h_{p}^{2}=0.2\right)$. There was no main effect or interaction with the factor hemispace, indicating that tapping speed did not differ when performed in the left or right hemispace (all $p$ 's $>0.4$ ).

Given the absence of a main effect of factor hemispace, and in order to reduce the number of tests and to increase statistical power, follow-up analyses were conducted after grouping the data

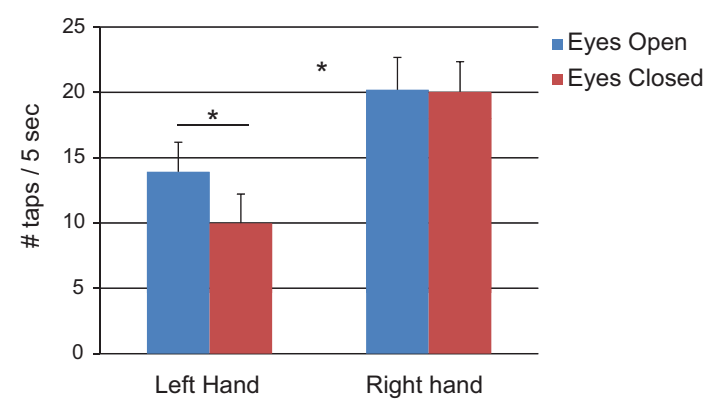

Fig. 3. Tapping rapidity evolution in $20 \mathrm{~s}$. Tapping speed is lower in the initiation part of the left hand with closed eyes (LH CE). Note the effect of presence of visual feedback. (20-s session was divided into four sections). LH OE: Left hand open eyes, LH CE: Left hand closed eyes, RH OE: Right hand open eyes and RH OE: Right hand open eyes.

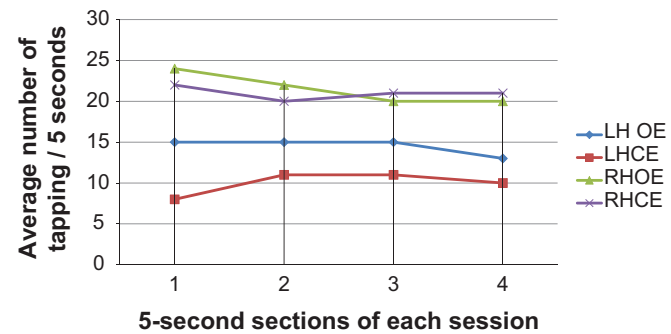

Fig. 4. Tapping speed for the right and left hand, with (blue) or without visual feedback (red). For the left but not right hand, tapping speed was higher with than without visual feedback. Standard deviations indicated. Asterisks: $p<0.001$. (For interpretation of the references to color in this figure legend, the reader is referred to the web version of this article.)

from the left and right hemispaces (Fig. 3). The two-way ANOVA with factors hand and visual feedback showed a main effect of hand $\left(f\left(1,140=465.5 ; p<0.001 ; h_{p}^{2}=0.8\right)\right.$, a main effect of visual feedback $\left(f\left(1,140=29.5 ; p<0.001 ; h_{p}^{2}=0.2\right)\right.$ and a interaction between these factors $\left(f\left(1,140=25 ; p<0.001 ; h_{p}^{2}=0.2\right)\right.$.

Post-hoc $t$-tests were conducted to test the influence of visual feedback on right- and left-hand tapping speeds separately. For the right hand, there was no evidence of a visual feedback effect. Tapping was no different with than without visual feedback $(t(70)=0.3 ; p=0.8)$. For the left hand, tapping was faster with than without visual feedback $(t(70)=7.6 ; p<0.001)$.

\subsection{Initiation speed}

There was a main effect of hand (i.e. faster initiation in right than left hand, $\left.f(1,44)=70.69, p<0.001, h_{p}^{2}=0.616\right)$, a main effect of visual feedback (i.e. faster initiation with open vs closed eyes, $\left.f(1,44)=479.4, p<0.001, h_{p}^{2}=0.916\right)$ and a significant hand $\times v i-$ sual feedback interaction on initiation speed which shows a greater improvement of tapping initiation in the presence of visual feedback with left hand right hand, $f(1,44)=44.3, p<0.001$, $h_{p}^{2}=0.502$ ). Fig. 4 summarizes these results.

\section{Discussion}

We reported a case of transitory akinesia following an ischemic CVA disconnecting the BG and SMA proper. This akinesia of the left hand impaired both initiation and speed of movements. The akinesia lasted two weeks and then recovered rapidly in less than a week.

During the symptomatic (akinesia) period, the patient underwent a finger-tapping task with left and right hands and showed a decrease in sustained rapidity of the left hand: he was unable to 
maintain a regular rhythm and to repeat the finger tapping with similar amplitude and fixed speed. However, we observed significant improvement in movement initiation and rapidity when the patient performed the finger-tapping experiment in the presence of visual feedback.

Akinesia recovered in two weeks. The regressive nature of the symptoms with an excellent motor outcome has been mentioned as a hallmark of SMA syndrome and SMA-basal ganglia disconnection (Kim, 2001; Kasasbeh et al., 2012; Krainik et al., 2001). Although the mechanism of rapid resolution of the symptoms is not fully understood, recruitment of the contralateral SMA and a preserved ipsilateral premotor area are considered to play key roles in this recovery (Bannur \& Rajshekhar, 2000). Lesion reconstruction in the MRI imaging in the present case has pointed to disconnection of the right SMA and right BG, with preservation of the other premotor-subcortical loops. This finding is consistent with previous lesion and functional studies; Krainik et al. (2001) discovered that in patients having undergone resection of the medial frontal lobe, the motor deficit occurs when the SMA is resected. The same result has been observed in a case of CVA involving the SMA (Marsden, 1989). In non-human primates, surprisingly, a milder spectrum of motor symptoms (from asymptomatic to clumsy and uncoordinated finger movements) has been reported in the first few weeks following unilateral or bilateral ablation of the SMA (Tanji, 1994).

The motor slowness present in the patient can be considered to be of premotor origin, rather than related to general or spatial attentional disorders. In particular, there were no criteria for a directional hypokinesia, a condition characterized by the inability to execute movements even with the unaffected arm in or toward the contralesional hemispace in patients with hemineglect (Coslett et al., 1990; Sapir et al., 2007). Indeed, the tapping task performed by the right and left hands in both ipsilateral and contralateral hemispaces was no different between the right and left hemispaces.

Rather, such impairment in speed of movement initiation and maintenance is a clinical feature of BG disorders, particularly Parkinson's disease. Such symptoms have also been described in the surgical resection of SMA (SMA syndrome), SMA lesions or lesions disconnecting SMA and BG. This can be explained by the fact that the main input projection to the SMA is derived from the BG. In addition, SMA and BG have been suggested to be involved in motor and perceptual timing tasks. Particularly, disconnection of BG output to the SMA due to an ACA infarction have been shown to produce Parkinsonian symptoms (tremor, rigidity, hypokinesia and akinesia) (Kim, 2001). Moreover, by describing a case of movement slowness and impaired action control following lesion in SMA, Gentilucci et al., 2000 proposed that the SMA proper may elaborate an initial force pulse for the execution of the motor act, which activates a cortical-subcortical loop, namely the spinal cord, $\mathrm{BG}$, posterior SMA and primary motor cortex for motor execution. In MPTP-treated monkeys, data dopamine depletion is associated with decreased SMA neural activity to visual cues, suggesting a critical role of SMA hypoactivity in preventing movement initiation and in akinesia Escola et al. (2003).

Movement initiation was significantly slower in the affected hand while the visual feedback was absent. This slowness was compensated with open eyes (i.e. there was no difference of tapping speed between the first and other three segments of the 20-s tapping period). The role of SMA proper-BG pathway in the movement initiation process was proposed by Gentilucci et al. (2000).The most important result is the decrease of the neurological deficit by visual compensation. The SMA is known to be more activated when performing the movements in the absence of external cues (e.g., visual feedback). While performing repetitive finger-movement tasks, i.e., driven by automatic internal control and not by visual feedback, the neural activity has been shown to increase in the SMA and BG. Consistently, in Parkinson's disease patients where the BG output to the SMA is impaired, the guiding of movement relies more on visual feedback; as a result, external pacing and visual cues are typically shown to accelerate initiation and to increase size of movement (Cunnington et al., 1996; Jahanshahi et al., 1995; Ogawa et al., 2006; Oliveira et al., 1997). This external pacing can compensate for the decreased SMAinduced automatic motor activation. Several activation studies further showed that the SMA and BG are under-activated during visually-guided movements. In contrast, the premotor area receives visual inputs from the posterior parietal cortex (Cunnington et al., 1996) and is involved in performing visually-guided movements. Therefore, the improvement of akinesia in presence of visual feedback can also be explained by the function of the premotor structures in movement preparation and initiation (Goldberg, 1985).

Previous studies report that external cues improve specific aspects of movement performance. Most notably, Freeman, Cody, \& Schady (1993) studied Parkinson's disease patients and showed that finger tapping rate increased in the presence of lowintermediate frequencies $(2-3 \mathrm{~Hz})$ of auditory "clicks". As another example, Oliveira et al. (1997) report that use of guiding lines and dots can increase the size of writing in Parkinson's disease patients in comparison to writing without cueing. In contrast to these studies in which an external feedback independent on the participant was used, we showed that visual information alone also improves the speed of movement initiation and maintenance.

\section{Conclusion}

Taken together, our behavioral data revealed that SMA-basal ganglia disconnection leads to a decrease in the speed of both movement initiation and maintenance. This akinesia is partly improved in the presence of visual feedback. This supports the available results on the role of SMA in performing movements in the absence of external cues.

\section{Acknowledgement}

This study supported by the Swiss National Science Foundation (PP00_1_128599/1 to SC and 325130-118362 to JMA). The authors thanks Ann Travis for providing language help.

\section{Appendix A. Supporting information}

Supplementary data associated with this article can be found in the online version at http://dx.doi.org/10.1016/j.neuropsychologia. 2013.08.004.

\section{References}

Bannur, U., \& Rajshekhar, V. (2000). Post operative supplementary motor area syndrome: Clinical features and outcome. British Journal of Neurosurgery, 14(3), 204-210.

Bohannon, R. (2003). Grip strength: A summary of studies comparing dominant and nondominant limb measurements. Perceptual and Motor Skills, 96, 728-730.

Boudrias, M., et al. (2006). Contrasting properties of motor output from the supplementary motor area and primary motor cortex in rhesus macaques. Cerebral Cortex, 16, 632-638

Boudrias, M., et al. (2010). Forelimb muscle representations and output properties of motor areas in the mesial wall of rhesus macaques. Cerebral Cortex, 20, 704-719.

Catalan, M. J., et al. (1998). The functional neuroanatomy of simple and complex sequential finger movements: A PET study. Brain, 121, 253-264.

Catalan, M. J., et al. (1999). A PET study of sequential finger movements of varying length in patients with Parkinson's disease. Brain, 122, 483-495.

Coslett, H., et al. (1990). Directional hypokinesia and hemispatial inattention in neglect. Brain, 113(2), 475-486. 
Council., M. R., Aids to the exaination of the peripheral nervous system., H.M.s.S. Office., editor 1976: London.

Cunnington, R., Bradshaw, J. L., \& Iansek, R. (1996). The role of the supplementary motor area in the control of voluntary movement. Human Movement Science, 15 , 627-647.

Cunnington, R., et al. (2002). The preparation and execution of self-initiated and externally-triggered movement: A study of event-related fMRI. NeuroImage, 15 (2), 373-385.

D'Ostilio, K., et al. (2012). Evidence for a role of a cortico-subcortical network for automatic and unconscious motor inhibition of manual responses. PLoS One, 7, 10

Escola, L., et al. (2003). Disruption of information processing in the supplementary motor area of the MPTP-treated monkey: A clue to the pathophysiology of akinesia? Brain, 126(Pt 1), 95-114.

Freeman, J. S., Cody, F. W., \& Schady, W. (1993). The influence of external timing cues upon the rhythm of voluntary movements in Parkinson's disease. Journal of Neurology, Neurosurgery \& Psychiatry, 56(10), 1078-1084.

Fries, W. (1985). Inputs from motor and premotor cortex to the superior colliculus of the macaque monkey. Behavioural Brain Research, 18(2), 95-105.

Gentilucci, M., et al. (2000). Impaired control of an action after supplementary motor area lesion: A case study. Neuropsychologia, 38(10), 1398-1404.

Goldberg, G. (1985). Supplementary motor area structure and function: Review and hypotheses. Behavioral and Brain Sciences, 8(4), 567-588.

Halsband, U., et al. (1992). The role of premotor cortex and the supplementary motor area in the temporal control of movement in man. Brain, 116(1), 243-266.

He, S., Dum, R., \& Strick, P. (1995). Topographic organization of corticospinal projections from the frontal lobe: Motor areas on the medial surface of the hemisphere. Journal of Neuroscience, 15, 3284-3306.

Herrero, M., Barcia, C., \& Navarro, J. (2002). Functional anatomy of thalamus and basal ganglia. Child's Nervous System, 18, 386-404.

Jahanshahi, M., et al. (1995). Self-initiated versus externally triggered movements. I. An investigation using measurement of regional cerebral blood flow with PET and movement-related potentials in normal and Parkinson's disease subjects. Brain, 118(4), 913-933.

(1992). Impaired activation of the supplementary motor area in Parkinson's disease is reversed when akinesia is treated with apomorphine. Annals of Neurology, 32(6), 749-757.
Kasasbeh, A. S., et al. (2012). Characterization of the supplementary motor area syndrome and seizure outcome after medial frontal lobe resections in pediatric epilepsy surgery. Neurosurgery, 70(5), 1152-1168.

Kim, J. S. (2001). Involuntary movements after anterior cerebral artery terittory infarction. Stroke, 32, 258-261.

Krainik, A., et al. (2001). Role of the supplementary motor area in motor deficit following medial frontal lobe surgery. Neurology, 57(5), 871-878.

Luppino, G., et al. (1993a). Corticocortical connections of area F3 (SMA-proper) and area F6 (pre-SMA) in the macaque monkey. The Journal of Comparative Neurology, 338(1), 114-140.

uppino, G., et al. (1993b). Corticocortical connections of area F3 (SMA-proper) and area F6 (pre-SMA) in the macaque monkey. Journal of Comparative Neurology. $338,114-140$.

Maier, M., et al. (2002). Differences in the corticospinal projection from primary motor cortex and supplementary motor area to macaque upper limb motoneurons: An anatomical and electrophysiological study. Cerebral Cortex, 12, 281-296.

Marsden, C. (1989). Slowness of movement in Parkinson's disease. Movement Disorders, 4(Suppl. 1), s26-s37.

Ogawa, K., Inui, T., \& Sugio, T. (2006). Separating brain regions involved in internally guided and visual feedback control of moving effectors: An event-related fMR study. NeuroImage, 32(4), 1760-1770.

Okano, K. (1992). Temporal priority of premotor cortex over nearby areas in receiving visual cues in primates. Neuroreport, 3(5), 389-392.

Oliveira, R. M. et al. (1997). Micrographia in Parkinson's disease: The effect of providing external cues. Journal of Neurology, Neurosurgery, and Psychiatry, 63, 429-433.

Orgogozo, J., \& Larsen, B. (1979). Activation of the supplementary motor area during voluntary movement in man suggests it works as a supramotor area. Science. 206(4420), 847-850.

Roland, P., et al. (1980). Supplementary motor area and other cortical areas in organization of voluntary movements in man. Journal of Neurophysiology, 43(1), $118-136$.

Sapir, A., et al. (2007). Anatomical correlates of directional hypokinesia in patients with hemispatial neglect. Journal of Neuroscience, 27(15), 4045-4051.

Tanji, J. (1994). The supplementary motor area in the cerebral cortex. Neuroscience Research, 19, 251-268. 